\title{
The Hedgehog signaling pathway in ovarian teratoma is stimulated by Sonic Hedgehog which induces internalization of Patched
}

\author{
MAJA SABOL $^{1}$, DIANA CAR ${ }^{1}$, VESNA MUSANI $^{1}$, PETAR OZRETIC $^{1}$, \\ SLAVKO ORESKOVIC $^{2}$, IGOR WEBER ${ }^{3}$ and SONJA LEVANAT ${ }^{1}$ \\ ${ }^{1}$ Division of Molecular Medicine, Rudjer Boskovic Institute, 10002 Zagreb; \\ ${ }^{2}$ Department of Obstetrics and Gynecology, School of Medicine, University of Zagreb, 10000 Zagreb; \\ ${ }^{3}$ Division of Molecular Biology, Rudjer Boskovic Institute, 10002 Zagreb, Croatia
}

Received April 4, 2012; Accepted May 29, 2012

DOI: 10.3892/ijo.2012.1554

\begin{abstract}
The Hedgehog-Gli (Hh-Gli) signaling pathway was examined in ovarian dermoids, which show characteristics of both tumors and developmental malformations. Dermoids are classified as mature teratomas that present differentiation into various tissues, mostly epidermal elements such as glands, multilayered epithelium, hair follicles and occasionally bone and cartilage. Their development is attributed to aberrant meiosis of germinal cells within the ovary. We showed activation of the Hh-Gli signaling in ovarian dermoid primary cultures. Cyclopamine treatment slows down cell proliferation, while the Sonic Hedgehog (Shh) protein stimulates cell proliferation and induces internalization of the Patched (Ptch) protein, which accumulates in the form of granules in the cytoplasm, colocalized with the Shh protein. Cyclopamine treatment decreases Gli1 localization in the nucleus compared to non-treated cells. Based on our observations, the mechanism of Hedgehog activation in the ovarian dermoids could be the ligand-dependent autocrine pathway, which can also be stimulated by paracrine signals.
\end{abstract}

\section{Introduction}

The Hh-Gli signaling pathway plays a crucial role in embryogenesis, but it is mostly inactive in adult tissues under normal conditions. The signaling transduction is initialized by the ligand Hedgehog with three different isoforms in humans: the Sonic Hedgehog (Shh), the Indian Hedgehog (Ihh) and the Desert Hedgehog (Dhh), which bind to the transmembrane receptor Patched (Ptch), inducing their internalization and

Correspondence to: Dr Sonja Levanat, Laboratory for Hereditary Cancer, Division of Molecular Medicine, Rudjer Boskovic Institute, Bijenicka cesta 54, 10002 Zagreb, Croatia

E-mail: levanat@irb.hr

Key words: hedgehog signaling, ovarian teratoma, cyclopamine, gene expression, confocal microscopy translocation of Smoothened (Smo) to the membrane. A cytoplasmatic cascade of phosphorylation events leads to activation of the transcription factor Gli, which translocates to the nucleus and initializes target gene transcription (1). The pathway activity is associated with the formation of various tissues and organs: neural tube, limbs, gastrointestinal system, lung, hair follicles, cartilage and bone (2), pancreas (3) and ganglia (4). In the adult organism it is associated with somatic stem cells, for example, breast (5), neural (6), lung (7), skin (8) and erythropoietic cells (9). When deregulated, it can lead to various tumors, for example, basocellular carcinoma, melanoma, trychoepithelioma, rhabdomyosarcoma, digestive tract tumors, prostate tumors, small-cell lung cancer, squamous lung cancer, pancreatic cancer, breast cancer and ovarian cancer (10).

The role of Hh-Gli signaling in ovarian development and malignancy has been explored by several groups. It has been shown that the pathway is involved in embryonic development and maturation of Drosophila ovaries, while in the adult fly it is involved in the division of stem cells giving rise to follicles (11). In mammals, the Hh-Gli pathway is also involved in embryonic development of the ovary, and in the adult organism components of the pathway are expressed in reproductive tissues (12). In the adult ovary, the Hh-Gli signaling is active in the granulose cells surrounding the follicle, and it can be additionally activated by exogenous Shh protein (13).

Ovarian carcinomas express Hh-Gli pathway genes, and in ovarian cancer cell lines cyclopamine, a known Hh-Gli pathway inhibitor, causes G1 cell cycle arrest (14). Also, ovarian carcinomas frequently show loss of one allele of the PTCHI gene (15), and ovarian teratomas show increased methylation of the $P T C H 1$ promoter (16).

Ovarian dermoids are mature teratomas which often contain various differentiated tissues, for example glandular tissue, multilayered epithelium, hair follicles, bone, teeth and cartilage. Their development is attributed to aberrant meiosis of germinal cells within the ovary. Depending on the phase of meiosis during which the errors occur, the teratomas are roughly divided into 5 subtypes (17). Reports on the malign transformation of teratomas into carcinomas mostly describe squamous cell carcinoma, melanoma, adenocarcinoma, 
neuroectodermal tumors, glioblastoma and sarcoma (18). Such events are rare; they occur in postmenopausal women in up to $2 \%$ of all teratomas, and most frequently progress into squamous cell carcinoma (19).

Our lab has been studying the mechanisms by which the Ptch protein takes part in the Hh-Gli signal transduction pathway and how aberrations in the pathway can lead to malignancy. Our previous findings showed the role of the PTCH1 gene in ovarian dermoids (20) and its association with Cyclin D (21), indicating the role of Hh-Gli signaling in the pathogenesis of this heterogenic neoplasia.

\section{Materials and methods}

Immunohistochemical and immunofluorescent staining. Paraffin tissue slides were routinely prepared in the Department of Obstetrics and Gynecology, School of Medicine, Zagreb. For immunohistochemical staining, tissues were deparaffinized, endogenous peroxidase was blocked, the slides were heated in epitope-retrieval solution for $5 \mathrm{~min}$ at $85^{\circ} \mathrm{C}$ to enable correct epitope folding, and stained using the following antibodies diluted 1:100: goat polyclonal anti-Ptch (Santa Cruz Biotechnology Inc., Santa Cruz, CA, USA; sc-6147) (22), rabbit polyclonal anti-Smo (Santa Cruz; sc-19943) (23), rabbit polyclonal anti-Gli1 (Santa Cruz; sc-20687) (24) and rabbit polyclonal anti-Hh (Santa Cruz; sc-9024) (13). Secondary antibodies were bovine anti-goat-HRP and bovine anti-rabbitHRP, used at 1:100 dilution. For negative control, slides were treated in the same way omitting the primary antibody step. For positive control, parallel staining was performed on squamous cell carcinoma slides which were positive for Ptch, Smo, Gli1 and Shh in our previous study (25).

For immunofluorescence, cells were seeded on glass coverslips and treated with $7.5 \mu \mathrm{M}$ cyclopamine or $3 \mathrm{ng} / \mu \mathrm{l} \mathrm{Shh}$ protein. After $24 \mathrm{~h}$, cells were fixed using 3.5\% paraformaldehyde solution, permeabilized with methanol, heated in epitope-retrieval solution for $5 \mathrm{~min}$ at $85^{\circ} \mathrm{C}$ to enable correct epitope folding, and stained with the same primary antibodies used for immunohistochemistry, using the same dilutions. Secondary antibodies were conjugated with either Texas Red (TR) or Fluorescein (FITC), used at 1:100 dilution: donkey anti-goat-TR (Santa Cruz; sc-2783), mouse anti-goat-FITC (Santa Cruz; sc-2356), donkey anti-rabbit-TR (Santa Cruz, sc-2784), donkey anti-mouse-FITC (Santa Cruz; sc-2099) and nuclei were stained with DAPI. For negative control, slides were treated in the same way omitting the primary antibody step.

Mutation detection. The PTCHI gene was divided into 24 PCR products and analyzed using high-resolution melting analysis (26), followed by sequencing in both directions (27-29). For the $S M O$ gene, only exons 9 and 10 were analyzed in the same manner (30).

Cell culture experiments. Dermoid tissue collected during surgery was stored for a maximum of 2-3 h in serum-free MEM culture medium at $4^{\circ} \mathrm{C}$. For primary culture, tissue was washed briefly in trypsin, and then cut into small fragments and digested in $5 \mathrm{ml}$ trypsin overnight at $37^{\circ} \mathrm{C}$ and $5 \% \mathrm{CO}_{2}$. The following day the cells were resuspended and plated in several $10 \mathrm{~cm}$ petri dishes in MEM supplemented with $20 \%$ human serum (HS). Dishes were regularly checked for cell colonies, which usually appeared after a week, approximately. Individual colonies were collected and grown separately, as separate clone lines.

Ten established primary clone lines and two ovarian cancer cell lines (COLO-704 and SkOv-3, a kind gift from Dr K. Rajalingam) were grown in MEM supplemented with $10 \%$ HS or $0.5 \%$ HS. Cells were treated with $7.5 \mu \mathrm{M}$ cyclopamine (Toronto Research Chemicals), $7.5 \mu \mathrm{M}$ tomatidine (Sigma-Aldrich, St. Louis, MO, USA), or $3 \mathrm{ng} / \mu \mathrm{l}$ Shh protein (a kind gift from Dr A. Kenney) for 24, 48 or 72 h. RNA was extracted using TRIzol reagent (Invitrogen, Carlsbad, CA, USA) and proteins were extracted by standard methods. MTT assay was performed as per the manufacturer's instructions. For western blot analysis, the primary antibodies for Ptch, Smo, Gli1 and Hh were the same ones that were used for immunofluorescent staining, as well as goat anti-Actin (Santa Cruz; sc-1616). Secondary antibodies were bovine anti-goat-HRP and bovine anti-rabbit-HRP, and the signal was detected using SuperSignal West Pico (Pierce Biotechnology Inc., Rockford, IL, USA; \#34077).

Quantitative real-time PCR ( $q R T-P C R)$. One microgram of total RNA was reverse transcribed using TaqMan Reverse Transcription Reagents (Applied Biosystems). Quantitative real-time PCR was performed with the following primers: ARP F, 5'-GGCACCATTGAAATCCTGAGTGATGTG-3' and $A R P$ R, 5'-TTGCGGACACCCTCCAGGAAGC-3'; $P T C H$ F, 5'-TCCTCGTGTGCGCTGTCTTCCTTC-3' and $P T C H$ R, 5'-CGTCAGAAAGGCCAAAGCAACGTGA-3'; $S M O \mathrm{~F}$, 5'-CTGGTACGAGGACGTGGAGG-3' and $S M O$ R, 5'-AGG GTGAAGAGCGTGCAGAG-3'; GLI1 F, 5'-GCCGTGT AAAGCTCCAGTGAACACA-3' and GLII R, 5'-TCCCACT TTGAGAGGCCCATAGCAAG-3'; $S H H$ F, 5'-GAAAGC AGAGAACTCGGTGG-3' and $S H H$ R, 5'-GGTAAGT GAGGAAGTCGCTG-3' (25); $S U F U$ F, 5'-AACAGCAA ACCTGTCCTTCC-3' and SUFU R, 5'-TCAGATGTACG CTCTCAAGC-3' (31); $\beta$-catenin F, 5'-GCGTGGACAA TGGCTACTCA-3' and $\beta$-catenin $\mathrm{R}, 5^{\prime}$-CCGCTTTTCT GTCTGGTTCC-3' (32). The reaction was performed in a $10 \mu \mathrm{l}$ reaction using IQ SYBR-Green supermix (Bio-Rad), under the following conditions: initial denaturation at $95^{\circ} \mathrm{C}$ for $3 \mathrm{~min}$, and 40 cycles of $95^{\circ} \mathrm{C}$ for $15 \mathrm{sec}$ and $61^{\circ} \mathrm{C}$ for $1 \mathrm{~min}$. Melting curve of each product was examined after each real-time PCR experiment to verify their specificity, and expression was normalized using the $A R P$ housekeeping gene. Relative gene expression was calculated using the $2^{-\Delta \Delta \mathrm{Ct}}$ formula, with RNA from the normal ovary used as the reference. Normal ovary sample was a pool of 9 RNA samples from healthy ovaries, taken during surgery at the Department of Obstetrics and Gynecology from patients operated for reasons other than malignant transformation. All patients gave their informed consent before the samples were obtained, and the samples were collected with the approval of the hospital's Ethics Committee (No 01-600/34-1-2007) in accordance with the Health Care Laws of the Republic of Croatia (NN121/03).

Confocal microscopy. Laser scanning confocal microscopy was performed on the Leica TCS SP2 AOBS instrument. 
FITC emission was excited with the $488 \mathrm{~nm}$ laser and detected in the 499-576 $\mathrm{nm}$ range, whereas TR emission was excited with the $543 \mathrm{~nm}$ laser and detected in the 605-712 $\mathrm{nm}$ range. DAPI emission was excited with the $405 \mathrm{~nm}$ laser and detected in the 413-466 $\mathrm{nm}$ range. In order to prevent bleed-through of the FITC emission into the TR channel, images in the two channels were recorded sequentially. Cross-excitation of the FITC fluorophore by the green $543 \mathrm{~nm}$ laser was eliminated by adjusting the imaging conditions.

\section{Results}

Hh-Gli signaling pathway proteins can be detected in ovarian dermoids. Ovarian dermoids show a high level of differentiation, with many structures typical for skin (epithelium, mucous glands, serous glands), and stromal and cortical regions of ovaries. Immunohistochemical staining of dermoid tissue sections revealed the presence of the main components of the Hh-Gli signaling pathway (Fig. 1). Ptch intensively stains the epithelium and many types of glandular tissue present in the sections, but not the connective tissue. It is mostly localized to the cell membrane, but in many cells it is also localized close to the nucleus (Fig. 1, insets). Smo is typically found in the same regions as Ptch. Staining of Gli1 is weak and mostly limited to the basal layer of the epithelium, but also within the oocyte in the follicles. Hh protein shows mostly epithelial staining, with the strongest intensity in the basal layer, but it is also localized to oocytes in the follicles and to zona pellucida in the secondary follicles.

No PTCHI or SMO mutations are detected in ovarian dermoids. To test whether the pathway activation is caused by inactivating mutations in the $\mathrm{PTCH} 1$ gene or activating mutations in the $S M O$ gene, the entire coding region of PTCH1 including its promoter, and exons 9 and 10 of SMO were examined for mutations. No mutations were found in the SMO hot-spot region (exons 9 and 10). In the PTCHI gene only three known polymorphisms were detected and all three were in heterozygous form. Polymorphisms were detected in exon 2 (c.318C > T), exon 12 (c.1665T $>$ C) and intron 15 (c.2560IVS+9G $>$ C). The two exonic polymorphisms cause no change in the amino acid sequence, and all three polymorphisms are well documented (Patched Mutation Database www.cybergene.se/PTCH/).

Primary cultures derived from ovarian dermoids have a limited lifespan. Ten distinct clone lines were established from two dermoids, 5 lines from each sample. These cultures were established and maintained in MEM supplemented with $10 \%$ human serum (HS) to match the conditions in vivo as closely as possible. Developed primary lines are vimentinpositive, most likely mesenchymal in origin, with fibroblast morphology. Primary cultures had a limited lifespan and started to show morphology typical of 'old' cells at approximately the 10th passage (elongated shape, vacuolization, slowed division), and usually died out by the 15 th passage. All experiments were performed at a passage number $<10$.

Hh-Gli signaling pathway expression in ovarian dermoid primary cultures. mRNA expression was detected for $\mathrm{PTCH}$,

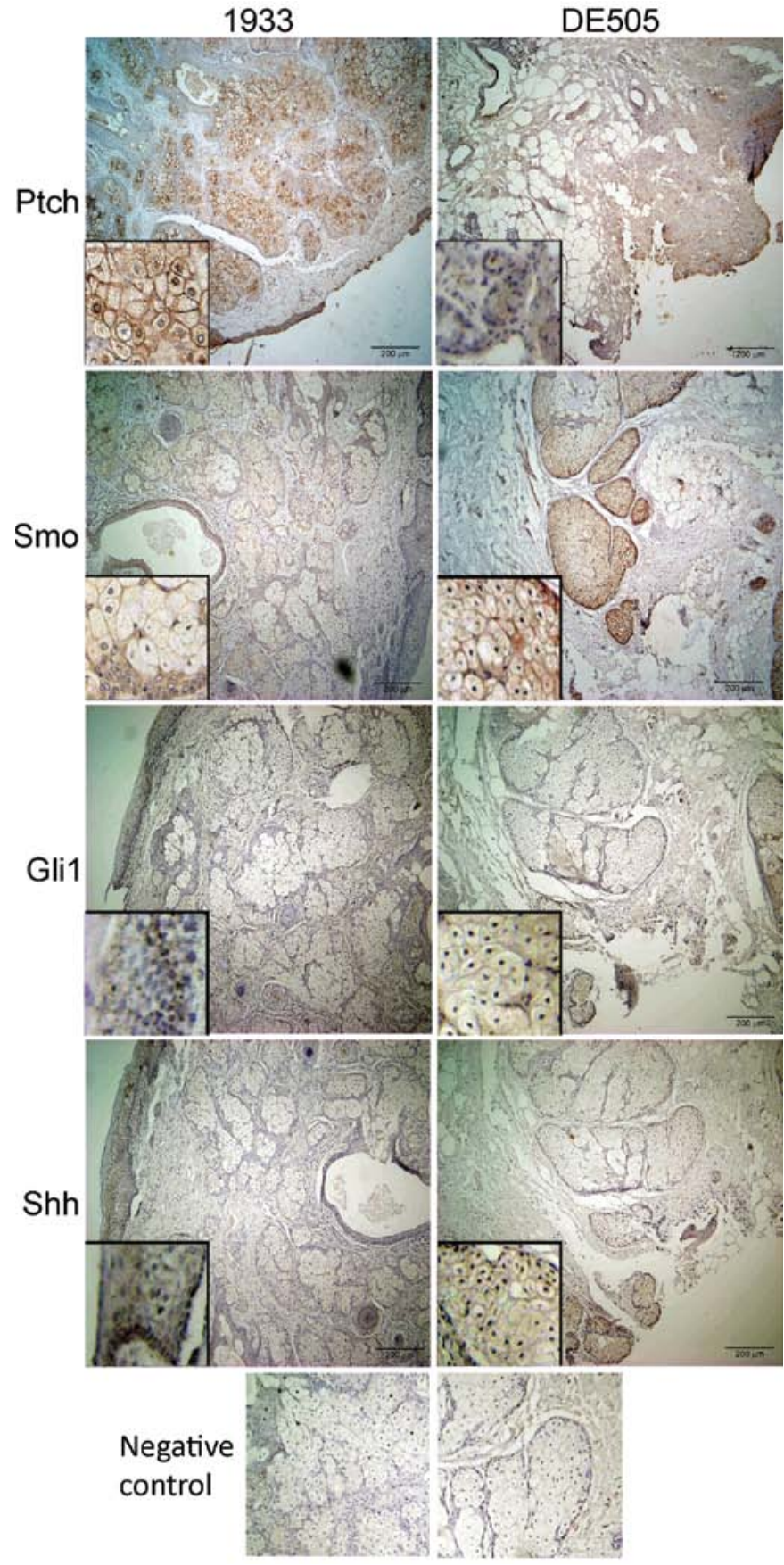

Figure 1. Immunohistochemical staining of the Ptch, Smo, Gli1 and Hh proteins in ovarian dermoid tissues, marked 1933 and DE505. Ptch and Smo are clearly stained on the cell membrane in the epithelium and glandular tissue, Gli1 shows a weak staining in the epithelium and oocytes, and the $\mathrm{Hh}$ staining pattern is very similar to Gli1.

SMO, GLII, SUFU, and $\beta$-catenin in all ten clone lines. SHH expression was not detectable after 40 cycles of quantitative real-time PCR in 5/10 clone lines. Since the healthy ovarian tissue shows no expression of Hh-Gli pathway proteins when examined by immunohistochemistry (14), we examined the gene expression levels in the healthy ovary and compared them to gene expression levels in dermoid cultures. PTCH1 and GLII expression, which are considered major markers of pathway activity, were upregulated in the dermoids compared to the normal ovary. PTCH1 levels were 40-100-fold higher in the dermoid than in the normal ovary, while GLII was not 


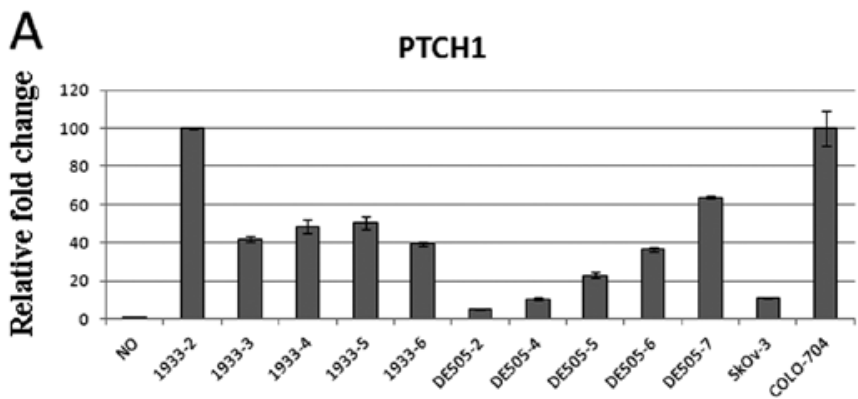

C

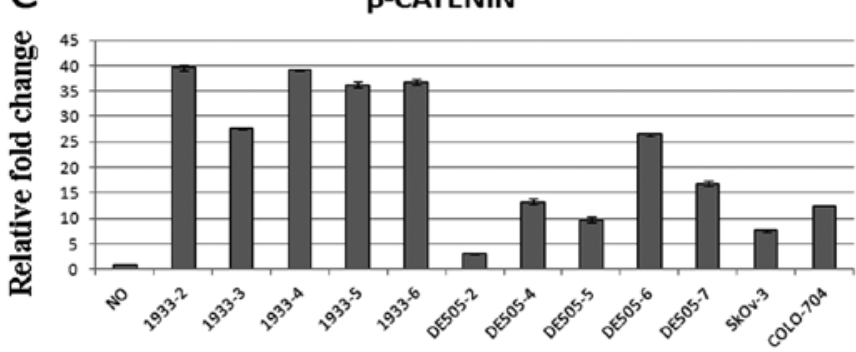

Figure 2. Relative expression of (A) PTCH1, (B) GLI1 and (C) $\beta$-catenin in 10 ovarian dermoid clone lines (1933-2 to -6, and DE505-2 to -7) and two ovarian carcinoma cell lines (SkOv-3 and COLO704) compared to the normal ovary (NO). Relative fold change was calculated using the $2^{-\Delta \Delta C t}$ formula.

detectable in the normal ovary, but was regularly detected in the dermoids with variations between the clone lines. $\beta$-catenin, which is regulated by the Hh-Gli signaling (33), was 25-40-fold more strongly expressed in the dermoid lines compared to the normal ovary (Fig. 2). Similar expression levels of the tested genes were also detected in the ovarian carcinoma cell lines SkOv-3 and COLO-704, which are also shown in Fig. 2.

Hh-Gli pathway proteins were also detected by western blot analysis. The Ptch protein was regularly detected in all clone lines. Smo and Gli1 expression was weaker than that of Ptch.

The Hh antibody we used shows reactivity to all three $\mathrm{Hh}$ proteins (Shh, Dhh, Ihh). In our experiments we detected one or two bands coresponding to approximately $50 \mathrm{kDa}$. Ovarian carcinoma cell lines showed a similar expression pattern of Hh-Gli pathway proteins. Cells grown in $0.5 \% \mathrm{HS}$, mimicking starvation, showed a far weaker signal for all pathway proteins, and Gli1 and Smo were often undetectable under these conditions (data not shown). Treatment with cyclopamine, tomatidine, or Shh protein did not change protein expression levels in any clone or cell line (Fig. 3).

Primary cultures show reactivity to cyclopamine and Shh protein. Since all clone lines derived from two tumors showed

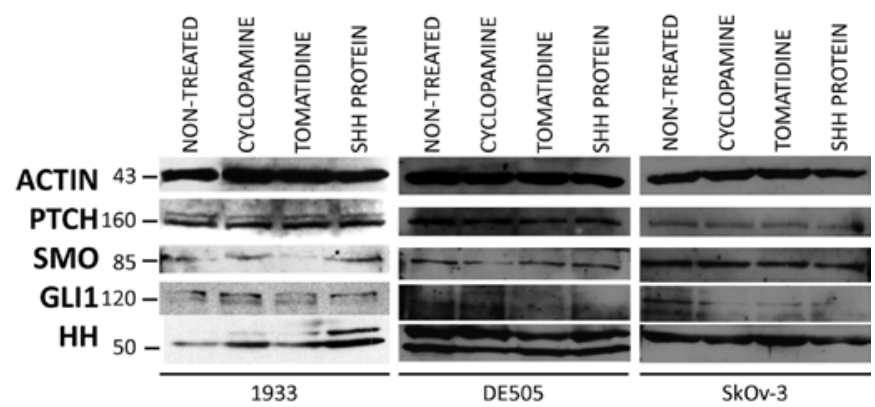

Figure 3. Western blot analysis of two different ovarian dermoid clone lines (one from each of the samples), as well as SkOv-3 ovarian cancer cell line, showing expression of Ptch, Smo, Gli1 and Hh proteins in non-treated cells, compared to cyclopamine, tomatidine or Shh protein-treated cells. No significant changes in protein levels were detected after any of the treatments.

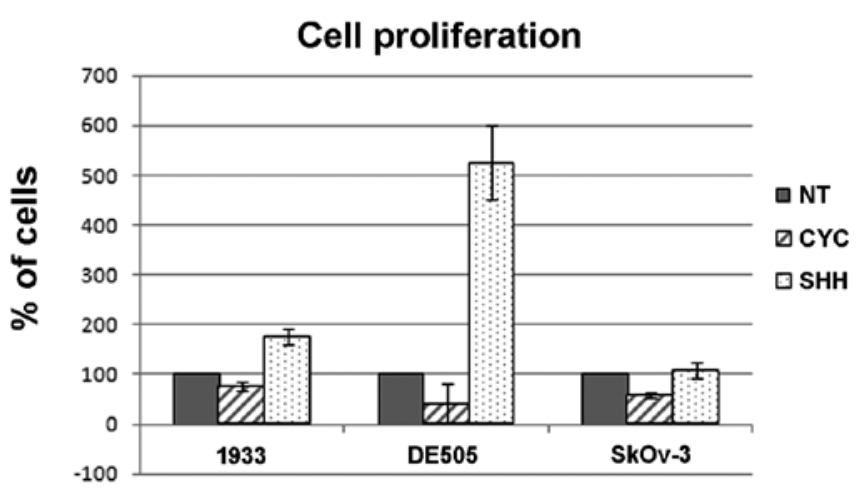

Figure 4. Cell proliferation was determined by MTT assay following treatment with $7.5 \mu \mathrm{M}$ cyclopamine or $3 \mathrm{ng} / \mu \mathrm{l}$ Shh protein for $72 \mathrm{~h}$. Primary cultures and $\mathrm{SkOv}-3$ cell line show a decrease in cell proliferation compared to non-treated cells, and primary cultures show an increase in proliferation after stimulation with the Shh protein.

expression of PTCH1, SMO, GLII and SUFU, one clone line from each tumor was used to determine the effect of the Smo inhibitor cyclopamine and responsiveness to Shh ligand. Cyclopamine-treated cells showed a significant delay in proliferation as determined by MTT proliferation assay (Fig. 4). On the other hand, treatment with the Shh protein caused an increase in cell proliferation compared to non-treated cells. The ovarian carcinoma cell line shows responsiveness to cyclopamine inhibition, but is not stimulated by the exogenous addition of the ligand. To test whether cyclopamine treatment affects cell cycle, as suggested by Chen et $a$ l (14), we tested cell cycle distribution after treatment with cyclopamine or Shh protein. Neither treatment affects the cell cycle distribution of these cells, suggesting a different mechanism of action from cell cycle control (data not shown).

Hh-Gli pathway proteins show altered localization after cyclopamine or Shh treatment. Cyclopamine and Shh treatment modulate localization of Ptch and Gli1 proteins suggesting this as the major regulatory step in Hh-Gli signaling in the dermoids. Their localization was altered in all tested clone lines in the same way. Fig. 5 shows the effect of cyclopamine (CYC) or Shh ligand (SHH) treatment on Ptch, Smo and Gli1 proteins compared to non-treated (NT) cells. 


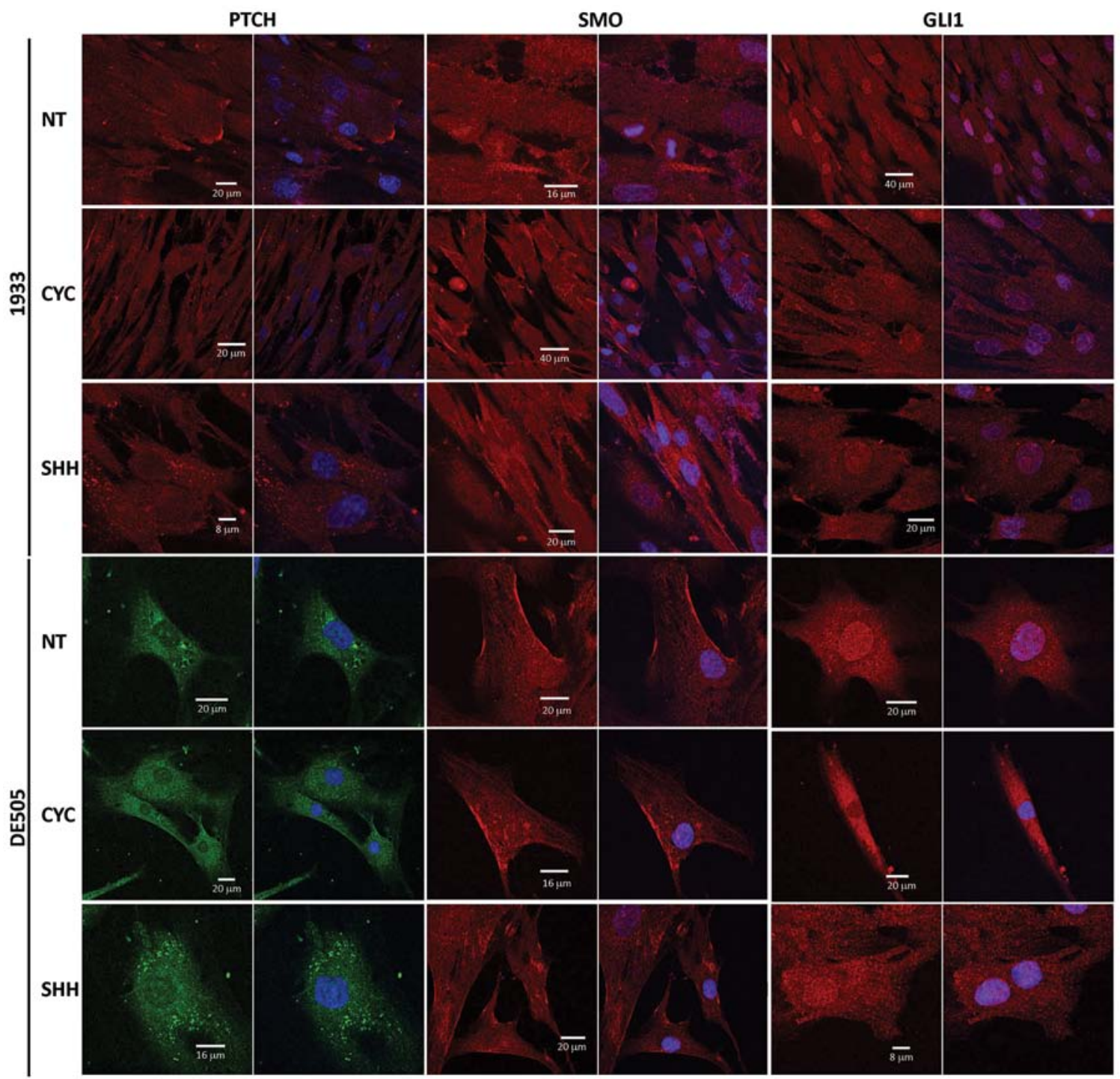

Figure 5. Cells derived from both dermoid samples were treated with $7.5 \mu \mathrm{M}$ cyclopamine or $3 \mathrm{ng} / \mu 1 \mathrm{Shh}$ protein, and protein localization was monitored with confocal microscopy. The Ptch protein is localized on the cell membrane and diffusely within the cell. Following Shh treatment it shows accumulation in the form of multiple dots in the cytoplasm. Following cyclopamine treatment, Ptch staining is diffused in the cytoplasm. The Smo protein is located on the cell membrane and in the cytoplasm, and its localization remains unchanged after either treatment. The Gli1 protein is located in the cytoplasm and more strongly in the nucleus, and after Shh treatment the signal is very similar to the unstimulated cells. Cyclopamine treatment reduces the intensity of Gli1 staining in the nucleus, which becomes less saturated than the surrounding cytoplasm.

The Ptch protein was regularly detected diffused through the cytoplasm and in patches at the cell membrane (Fig. 5, columns 1 and 2). After cyclopamine treatment the staining was more diffuse, while Shh treatment induced accumulation of dot-like structures in the cytoplasm. Smo protein was detected in the cytoplasm with a stronger staining of the cell membrane (Fig. 5, columns 3 and 4), and remained unchanged after either treatment. In non-treated cells the Glil protein was enriched in the nucleus compared to the cytoplasmic background. After treatment with cyclopamine, staining of the nucleus was decreased. Shh treatment had no significant effect on Gli1, since it was already localized in the nucleus and therefore active.

Treatment with the Shh protein had a remarkable effect on Ptch protein localization. After $24 \mathrm{~h}$ small bright dots of Ptch protein became visible in the cytoplasm, and after $48 \mathrm{~h}$ the dots increased in size and intensity. These cytoplasmatic Ptch aggregates were occasionally detected in untreated cells with lesser intensity (Fig. 6, NT), suggesting some baseline activity of the pathway, and were reduced in cyclopamine-treated cells (Fig. 6, CYC). When cells were stained with Ptch and Hh antibodies, it was clear that the aggregates contained both Ptch and Hh protein, suggesting their joint internalization after binding (Fig. 6, white arrows). A basal level of pathway activity is visible in non-treated cells, is significantly increased in Shh-treated cells and is not detectable in cyclopamine-treated cells.

Previous studies suggest that Hh-Gli signaling in mammals requires the primary cilia to effectively transduce the signal (reviewed in ref. 34). Therefore cells were stained for acetylated tubulin to check for primary cilia formation and possible colocalization with the pathway proteins. Despite our efforts, we were unable to find a large number of ciliated cells regardless of growth conditions, but occasionally cilia-like structures were detected (data not shown). 


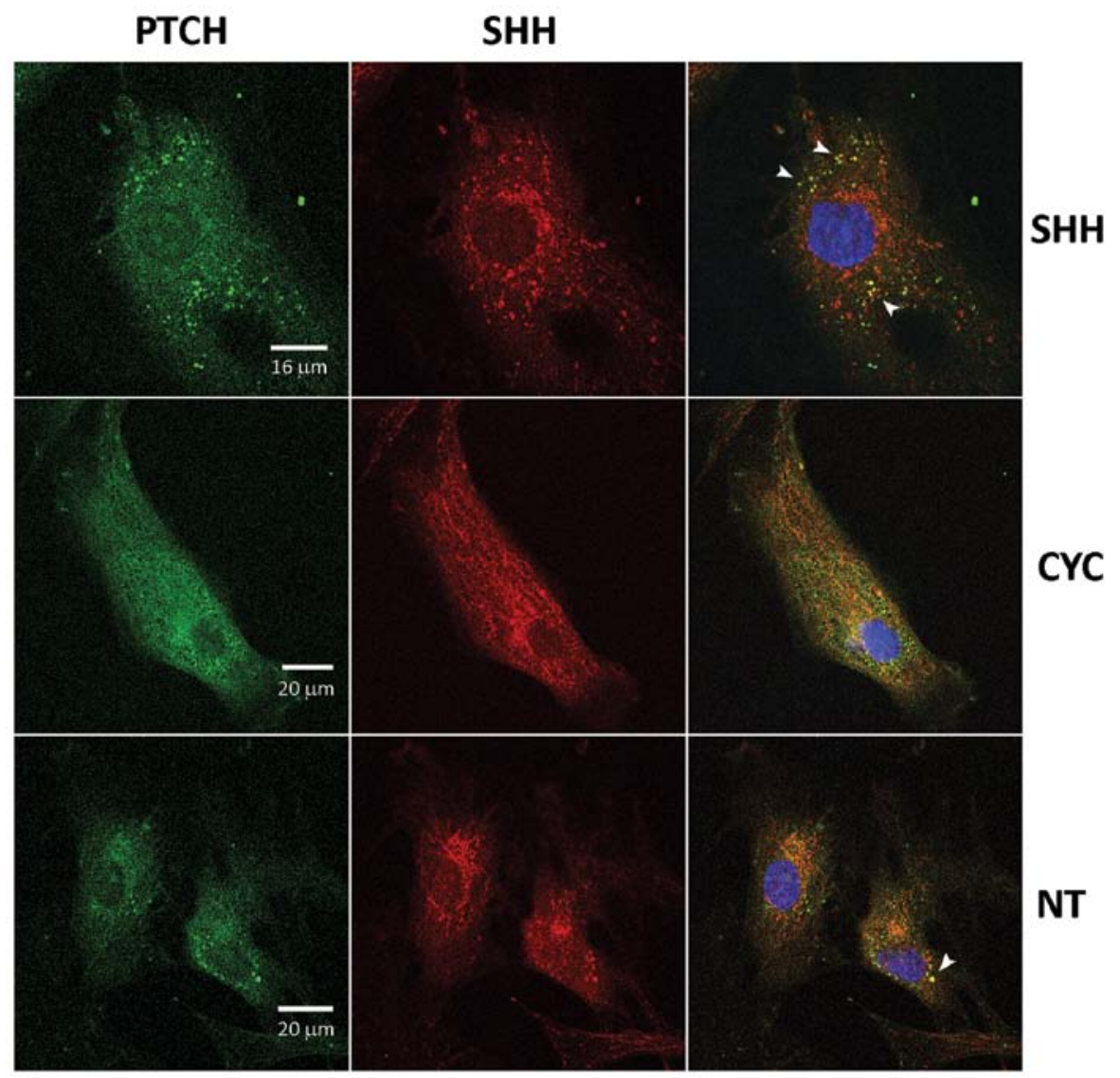

Figure 6. Dermoid cells were treated with $7.5 \mu \mathrm{M}$ cyclopamine or $3 \mathrm{ng} / \mu \mathrm{l}$ Shh protein, and Ptch and Hh protein colocalization was monitored with confocal microscopy. Hh protein is produced in ovarian dermoids and its staining is the strongest in the region surrounding the nucleus, most likely endoplasmatic reticulum. In the non-treated conditions (NT) a small number of Ptch cytoplasmatic dots is detected, suggesting a basal level of signaling activity. Upon Shh stimulation the number of these dots is dramatically increased and each of the dots also shows a signal for the Hh protein, suggesting their concomitant internalization. Cyclopamine-treated cells show only diffuse Ptch staining, while Hh production remains unaltered.

\section{Discussion}

Involvement of Hh-Gli signaling in ovarian carcinogenesis has been demonstrated $(14,15)$, but its detailed role in germ cell tumorigenesis has yet to be thoroughly examined. We used ovarian dermoids as they show characteristics of tumors and developmental malformations, and Hh-Gli signaling is involved in both processes. The structures which usually develop within dermoids belong to the tissue types that are controlled by Hh-Gli signaling in embryonic development, for example glandular tissue, hair follicles, bone, teeth and cartilage. Ovarian dermoids are classified as teratomas, a type of benign ovarian tumors, which contain differentiated elements derived from any of the three embryonic germ layers. The term 'teratoma' denotes the strange manifestations of benign or malignant tumors that possess the hallmarks of abnormal embryogenesis (35).

Our results show different levels of gene expression of Hh-Gli pathway genes in different clones from the same tumor tissue sample, confirming the known observation of heterogeneity of dermoid tissues. Developed primary lines are vimentin-positive, most likely mesenchymal in origin, with fibroblast morphology. However, it is unclear whether this vimentin-positive, cytokeratine-negative phenotype is the marker of a mesenchymal cell, or a consequence of the Hh-Gli pathway activation, which can induce epithelial-mesenchymal transition $(36,37)$. Also, ovarian cysts are one of the features of the Gorlin syndrome, which is caused by germline mutations in the $\mathrm{PTCH} 1$ gene. However, we did not detect any mutations in $\mathrm{PTCH} 1$ or SMO genes in the dermoid samples; only polymorphisms in exons 2 and 12 with no effect on amino acid sequence.

Results presented in this study demonstrate that the Hh-Gli signaling pathway is activated in the dermoid tissue, but not in the surrounding ovarian tissue. Major members of the pathway, namely Ptch, Smo, Gli1 and Hh, can be detected in the dermoid tissue, although Gli1 and Hh at lower levels than Ptch and Smo. This is not surprising since Gli1, as the transcription factor, plays a limiting role in controlling pathway activity. Hh-Gli signaling pathway genes PTCHI, SMO, GLII and SUFU are detected in all dermoid clone lines, with intensities similar to ovarian carcinoma cell lines. When dermoid primary cultures are compared to the normal ovary, PTCHI and GLIl, major markers of pathway activity, are strongly upregulated, as well as $\beta$-catenin, one of the Hh-Gli signaling targets. Gli2 and Gli3 do not appear to be relevant in Hh-Gli pathway control in ovarian dermoids, since their gene and protein expression was extremely weak and often undetectable. $S H H$ gene expression was detected in only five of the ten clone lines. When compared with western blot analysis, one to two Hh proteins 
are detected in ovarian dermoid primary cultures. Studies on mice have demonstrated that Dhh and Ihh are expressed in granulosa cells, pre-antral and antral follicles, and induce Hh-Gli signaling in the surrounding stroma (38). Therefore, the protein signal detected in our cells is more likely to be one of these two proteins.

Treatment with cyclopamine, a known inhibitor of the Hh-Gli pathway which binds to Smo and prevents downstream signaling, reduces cell proliferation in primary cultures and ovarian carcinoma cell line. On the other hand, Shh protein increases cell proliferation, and this effect has been previously demonstrated on mammalian neuronal precursors (39).

Protein localization demonstrated by confocal microscopy also corroborates previously published data: Gli1 is located in the cytoplasm and in the nucleus, whereas Ptch and Smo are located in the cytoplasm and on the cell membrane (10). Gli1 changes localization after cyclopamine treatment, showing a reduction of the signal in the nuclear interior.

It is well known that many membrane receptors are internalized and then degraded or recycled to the cell membrane (40). According to recent models, Ptch is also internalized upon Shh binding, allowing Smo to translocate to the cell membrane $(41,42)$. Indeed, our results provide evidence for this internalization process. Addition of Shh protein induces the signal transduction, which leads to increased Ptch internalization, and colocalization of Ptch and Shh proteins within these granules in the cytoplasm (Fig. 5).

It has been demonstrated that the primary cilia are required for Hh-Gli pathway regulation in mammalian development, where they act as a switch for turning the signaling on and off (34). However, the cilia are not required for the Drosophila Hh signaling, and the signaling events involving $\mathrm{SuFu}$ and Gli proteins are independent of cilia (43). Here, we detected structures that stain for acetylated tubulin, and resemble the primary cilia. However there was no enrichment of the Smo protein inside the cilia. Since the primary cilia are usually found on the surface of most growth-arrested and differentiated cells, it is not surprising that they were not detected more often in primary dermoid cells, since these were quite proliferative in culture. The role of primary cilia in tumorigenesis remains unclear, and published reports state that either the presence or the absence of cilia can enhance tumor growth, depending on the molecular context $(44,45)$. It has been reported that Hh-Gli pathway responsiveness correlates with the presence of the primary cilia in prostate development (46). However, when prostate cancer cell lines were examined under different growth conditions, there was no evidence of cilia formation (46). The cilia were also normally detected in the ovary, more specifically in the granulosa cells of the pre-antral and antral follicles (47). When cultured, these cells exhibit cilia only after serum starvation and growth arrest, while normal interphase cells show a complex cytoskeletal network but no primary cilia (47). This, as well as our data, suggests that the pathway may be regulated differently in normal development compared to cancer, and further investigation of these differences may lead to potential Hh-Gli pathway-targeting drugs.

Teglund and Toftgard (1) proposed that Hedgehogassociated tumors may have four different mechanisms of activation: the ligand-independent, the ligand-dependent autocrine, the ligand-dependent paracrine, and the ligand- dependent reverse paracrine mechanism. The dermoids express $\mathrm{Hh}$ ligand, and show increased proliferation and changes in protein localization consistent with pathway activity. A basal level of pathway activity can be seen in all the clone lines and this is greatly increased by addition of the Shh ligand. Based on our observations, the mechanism of Hedgehog activation in the ovarian dermoids could be the ligand-dependent autocrine pathway, which can also be stimulated by paracrine signals. The basal level of autocrine activity may keep the cells in a 'ready' state, prepared to react to outside stimulus, possibly by a signal from granulosa cells or developing follicles which are known to produce the $\mathrm{Hh}$ ligands. Since dermoids are considered to develop from aberrantly activated germinal cells within the ovary, their microenvironment which produces $\mathrm{Hh}$ ligands provides a proliferation signal and enables dermoid formation.

\section{Acknowledgements}

This study was funded by the Croatian Ministry of Science, Education and Sports, grant no. 098-0982464-2461. The authors wish to thank all the patients who participated in the study. We would also like to thank Dr A. Kenney for providing the Shh protein, Dr K. Rajalingam for the SkOv-3 and COLO-704 cells, Professor Dr B. Sarcevic for vimentin and cytokeratin characterization of clone lines, and L. Horvat, $\mathrm{BSc}$, for her assistance with the confocal microscopy.

\section{References}

1. Teglund $\mathrm{S}$ and Toftgård R: Hedgehog beyond medulloblastoma and basal cell carcinoma. Biochim Biophys Acta 1805: 181-208, 2010.

2. Jia J and Jiang J: Decoding the Hedgehog signal in animal development. Cell Mol Life Sci 63: 1249-1265, 2006.

3. Hebrok M: Hedgehog signaling in pancreas development. Mechanisms Dev 120: 45-57, 2003.

4. Masai I, Yamaguchi M, Tonou-Fujimori N, Komori A and Okamoto H: The hedgehog-PKA pathway regulates two distinct steps of the differentiation of retinal ganglion cells: the cell-cycle exit of retinoblasts and their neuronal maturation. Development 132: 1539-1553, 2005.

5. Kalirai $\mathrm{H}$ and Clarke RB: Human breast epithelial stem cells and their regulation. J Pathol 208: 7-16, 2006.

6. Stecca B and Ruiz i Altaba A: Brain as a paradigm of organ growth: Hedgehog-Gli signaling in neural stem cells and brain tumors. J Neurobiol 64: 476-490, 2005.

7. Watkins DN, Berman DM, Burkholder SG, Wang B, Beachy PA and Baylin SB: Hedgehog signaling within airway epithelial progenitors and in small-cell lung cancer. Nature 422: 313-317, 2003.

8. Zhou YX, Jia LW, Liu WM , Miao CL, Liu S, Cao YJ and Duan EK: Role of sonic hedgehog in maintaining a pool of proliferating stem cells in the human fetal epidermis. Hum Reprod 21: 1698-1704, 2006.

9. Detmer K, Thompson AJ, Garner RE, Walker AN, Gaffield W and Dannawi H: Hedgehog signaling and cell cycle control in differentiating erythroid progenitors. Blood Cells Mol Dis 34: 60-70, 2004.

10. Scales SJ and de Sauvage FJ: Mechanisms of Hedgehog pathway activation in cancer and implications for therapy. Trends Pharmacol Sci 30: 302-312, 2009.

11. Zhang Y and Kalderon D: Hedgehog acts as a somatic stem cell factor in the Drosophila ovary. Nature 410: 599-604, 2001.

12. Walterhouse DO, Lamm ML, Villavicencio E and Iannaccone PM: Emerging roles for hedgehog-patched-Gli signal transduction in reproduction. Biol Reprod 69: 8-14, 2003.

13. Russel MC, Cowan RG, Harman RM, Walker AL and Quirk SM: The hedgehog signaling pathway in the mouse ovary. Biol Reprod 77: 226-236, 2007. 
14. Chen X, Horiuchi A, Kikuchi N, Osada R, Yoshida J, Shizawa T and Konishi I: Hedgehog signal is activated in ovarian carcinomas, correlating with cell proliferation: its inhibition leads to growth suppression and apoptosis. Cancer Sci 98: 68-76, 2007.

15. Byrom J, Mudaliar V, Redman CW Jones P, Strange RC and Hoban PR: Loss of heterozygosity at chromosome 9q22-31 is a frequent and early event in ovarian tumors. Int J Oncol 24: 1271-1277, 2004.

16. Cretnik M, Musani V, Oreskovic S, Leovic D and Levanat S: The Patched gene is epigenetically regulated in ovarian dermoids and fibromas, but not in basocellular carcinomas. Int J Mol Med 19: 875-883, 2007

17. Surti U, Hoffner L, Chakravarti A and Ferrell RE: Genetics and biology of human ovarian teratomas. I. Cytogenetic analysis and mechanism of origin. Am J Hum Genet 47: 635-643, 1990.

18. Ulbright TM: Germ cell tumors of the gonads: a selective review emphsizing problems in differential diagnosis, newly appreciated, and controversial issues. Mod Pathol 18: S61-S79, 2005.

19. Bal A, Mohan H, Singh SB and Sehgal A: Malignant transformation in mature cystic teratoma of the ovary: report of five cases and review of the literature. Arch Gynecol Obstet 275: 179-182, 2007.

20. Levanat S, Pavelic B, Crnic I, Oreskovic S and Manojlovic S: Involvement of PTCH gene in various noninflammatory cysts. J Mol Med 78: 140-146, 2000

21. Levanat S, Kappler R, Hemmerlein B, Doring P, Musani V, Komar A, Orešković S, Pavelić B and Hahn H: Analysis of alterations of the PTCH1 signaling pathway in ovarian dermoids. Int J Mol Med 14: 793-799, 2004.

22. Yoshizaki A, Nakayama T, Naito S, Wen CY and Sekine I: Expressions of sonic hedgehog, patched, smoothened and Gli-1 in human intestinal stromal tumors and their correlation with prognosis. World J Gastroenterol 12: 5687-5691, 2006.

23. Liao X, Siu MK, Au CW, Wong ES, Chan HY, Ip PP, Ngan HY and Cheung AN: Aberrant activation of hedgehog signaling pathway in ovarian cancers: effect on prognosis, cell invasion and differentiation. Carcinogenesis 30: 131-140, 2009.

24. Regl G, Neill GW, Eichberger T, Kasper M, Ikram MS, Koller J, Hintner H, Quinn AG, Frischauf AM and Aberger F: Human GLI2 and GLI1 are part of a positive feedback mechanism in basal cell carcinoma. Oncogene 21: 5529-5539, 2002.

25. Leovic D, Sabol M, Ozretic P, Musani V, Car D, Marjanovic K, Zubcic V, Sabol I, Sikora M, Grce M, et al: Hh-Gli signaling pathway activity in oral and oropharyngeal squamous cell carcinoma. Head Neck 34: 104-112, 2012.

26. Cvok ML, Cretnik M, Musani V, Ozretic P and Levanat S: New sequence variants in BRCA1 and BRCA2 genes detected by high-resolution melting analysis in an elderly healthy female population in Croatia. Clin Chem Lab Med 46: 1376-1383, 2008.

27. Boutet N, Bignon YJ, Drouin-Garraud V, Sarda P, Longy M, Lacombe D and Gorry P: Spectrum of PTCH mutations in French patients with Gorlin syndrome. J Invest Dermatol 121: 478-481, 2003

28. Hahn H, Wicking C, Zaphiropoulous PG, Gailani MR, Shanley S, Chidambaram A, Vorechovsky I, Holmberg E, Unden AB, Gillies S, et al: Mutations of the human homolog of Drosophila patched in the nevoid basal cell carcinoma syndrome. Cell 85: 841-851, 1996.

29. Lench NJ, Telford EA, High AS, Markham AF, Wicking C and Wainwright BJ: Characterisation of human patched germ line mutations in naevoid basal cell carcinoma syndrome. Hum Genet 100: 497-502, 1997.

30. Xie J, Murone M, Luoh SM, Ryan A, Gu Q, Zhang C, Bonifas JM, Lam CW, Hynes M, Goddard A, et al: Activating Smoothened mutations in sporadic basal-cell carcinoma. Nature 391: 90-92, 1998.
31. Koch A, Waha A, Hartmann W, Milde U, Goodyer CG, Sorensen N, Berthold F, Digon-Sontgerath B, Kratzschmar J, Wiestler OD and Pietsch T: No evidence for mutations or altered expression of the Suppressor of Fused (SUFU) in primitive neuroectodermal tumours. Neuropath Applied Neurobiol 30: 532-539, 2004.

32. Wong SC, Lo SF, Cheung MT, Ng KO, Tse CW, Lai BS, Lee KC and Lo YM: Quantification of plasma $\beta$-catenin mRNA in colorectal cancer and adenoma patients. Clin Cancer Res 10: 1613-1617, 2004

33. Mullor JL, Dahmane N, Sun T and Ruiz i Altaba A: Wnt signals are targets and mediators of Gli function. Curr Biol 11: 769-773, 2001.

34. Veland IR, Awan A, Pedersen LB, Yoder BK and Christensen ST: Primary cilia and signaling pathways in mammalian development, health and disease. Nephron Physiol 111: 39-53, 2009.

35. Solter D: From teratocarcinomas to embryonic stem cells and beyond: a history of embryonic stem cell research. Nature Review Genet 7: 312-327, 2006.

36. Huber MA, Kraut N and Beug $\mathrm{H}$ : Molecular requirements for epithelial-mesenchymal transition during tumor progression. Curr Opin Cell Biol 17: 548-558, 2005.

37. Savagner P: The epithelial-mesenchymal transition (EMT) phenomenon. Ann Oncol 21 (Suppl 7): vii89-vii92, 2010.

38. Wijgerde M,Ooms M,Hoogerbrugge JW and Anton Grootegoed J: Hedgehog signaling in mouse ovary: Indian Hedgehog and Desert Hedgehog from granulosa cells induce target gene expression in developing theca cells. Endocrinology 146: 3558-3566, 2005.

39. Kenney AM and Rowitch DH: Sonic Hedgehog promotes $\mathrm{G}(1)$ cyclin expression and sustained cell cycle progression in mammalian neuronal precursors. Mol Cell Biol 20: 9055-9067, 2000.

40. Acconcia F, Sigismund S and Polo S: Ubiquitin in trafficking: the network at work. Exp Cell Res 315: 1610-1618, 2009.

41. Rohatgi R, Milenkovic L and Scott MP: Patched1 regulates Hedgehog signaling at the primary cilium. Science 317 : 372-376, 2007.

42. Lu X, Liu S and Kornberg TB: The C-terminal tail of the Hedgehog receptor Patched regulates both localization and turnover. Genes Dev 20: 2539-2551, 2006.

43. Chen MH, Wilson CW, Li YJ, Law KKL, Lu CS, Gacayan R, Zhang X, Hui CC and Chuang PT: Cilium-independent regulation of Gli protein function by Sufu in Hedgehog signaling is evolutionarily conserved. Genes Dev 23: 1910-1928, 2009.

44. Han YG, Kim HJ, Dlugosz AA, Ellison DW, Gilbertson RJ and Alvarez-Buylla A: Dual and opposing roles of primary cilia in medulloblastoma development. Nature Med 15: 1062-1065, 2009.

45. Wong SY, Seol AD, So PL, Ermilov AE, Bichakjian CK, Epstein EE Jr, Dlugosz AA and Reiter JF: Primary cilia can both mediate and suppress Hedgehog pathway-dependent tumorigenesis. Nature Med 15: 1055-1061, 2009.

46. Zhang J, Lipinski RJ, Gipp JJ, Shaw AK and Bushman W: Hedgehog pathway responsiveness correlates with the presence of primary cilia on prostate stromal cells. BMC Dev Biol 9: 50, 2009.

47. Teilmann SC, Byskov AG, Pedersen PA, Wheatley DN, Pazour GJ and Christensen ST: Localization of transient receptor potential ion channels in primary and motile cilia of the female murine reproductive organs. Mol Reprod Dev 71: 444-452, 2005. 